\title{
EVALUACIÓN DE COMPETENCIAS Y ACTITUDES VINCULADAS AL PROCESO DE TITULACIÓN EN ESTUDIANTES DE LICENCIATURA
}

\author{
José Manuel García Cortés, Ana Teresa Rojas Ramírez, Eduardo Arturo Contreras Ramírez, \\ Alan Alexis Mercado Ruíz y María del Socorro Contreras Ramírez \\ Facultad de Estudios Superiores Zaragoza, Universidad Nacional Autónoma de México
}

\section{Resumen}

Existe una tendencia actual donde los modelos educativos se organizan e implementan con base al concepto de competencias. Esto resalta la educación como un proceso de adecuación entre el profesional, la demanda del medio y necesidades sociales, con la finalidad de dar respuestas a problemáticas cotidianas y emergentes. Distintos autores expresan su preocupación respecto a que los estudiantes de pregrado deben de identificar claramente cuáles son las competencias con las que cuentan acorde a su perfil de egreso. Lamentablemente en muchos casos a pesar de obtener el grado en tiempo y forma, no se logra. Esta investigación pretende retomar el proceso de titulación de estudiantes de último año, como el escenario para identificar las competencias profesionales según su perfil de egreso y construir una herramienta que permita evaluarlas. Se trabajó en dos etapas: un estudio exploratorio con 20 egresados de la carrera de Psicología de FES Zaragoza UNAM, a quienes se les aplicó una entrevista semi-estructurada para identificar la competencias y actitudes aplicadas al proceso de titulación. Mediante una codificación cerrada se obtuvo una red semántica de 6 dimensiones. Con esta información, en la etapa 2, se construyó un instrumento dicotómico el cual fue aplicado a 434 estudiantes de último semestre. Se obtuvo una escala de 49 ítems distribuidos en 6 dimensiones con una validez de contenido por juicio de expertos $(\mathrm{k}=.94)$ y una confiabilidad total Split half de $\mathrm{R}=.681$ en su coeficiente de Spearman-Brown para longitudes desiguales, que permite evaluar la percepción de competencias y actitudes aplicadas al proceso de titulación.

Palabras claves: competencias, formación profesional, titulación, medición, psicometría

\begin{abstract}
There is a current trend where educational models are organized and implemented based on the concept of competencies. This stand out education as a process of adaptation between the professional, the demand of the environment and social needs, in order to respond to daily and emerging problems. Different authors express their concern that undergraduate students must clearly identify what competencies they have according to their graduation profile. Unfortunately, in many cases, despite obtaining the degree in a timely manner, it is not achieved. This research aims to take the graduation process of final year students, as a scenario to identify professional competencies according to their graduation profile and build a scale that allows evaluating them. This research was developed in two stages: an exploratory study with 20 graduates of the Psychology career of FES Zaragoza UNAM, to whom a semi-structured interview was applied to identify the skills and attitudes applied to the degree process. By a closed coding, a 6-dimensional semantic network was obtained. With that information, in stage 2 , a dichotomous instrument was built, which was applied to 434 final semester students. A scale of 49 items distributed in 6 factors was obtained with a content validity by expert judgment $(\mathrm{k}=.94)$ and a Split half total reliability of $\mathrm{R}=.681$ in its Spearman-Brown coefficient for unequal lengths, which allows evaluate the perception of competences and attitudes applied to the degree process.
\end{abstract}

Key words: competences, professional training, qualifications, measurement, psychometrics

Correspondencia

José Manuel García Cortés

Facultad de Estudios Superiores Zaragoza, Universidad Nacional Autónoma de México. México Email: jose.garcia@zaragoza.unam.mx 


\section{Introducción}

Actualmente se dispone de un amplio conocimiento sobre cómo las personas aprenden: existen datos irrefutables acerca de la manera en que se adquieren contenidos conceptuales, procedimentales y actitudinales. El aprendizaje de las competencias está muy alejado de lo que es del aprendizaje mecánico, implican mayor grado de significado y función posible, ya que para poder ser utilizadas deben tener sentido tanto la propia competencia como los conocimientos, habilidades, actitudes y valores implicadas en esta misma (Florentino, 2001).

A su vez, la sociedad actual se caracteriza por cambios acelerados: una serie de factores generacionales como el impacto de las tecnologías de la información y comunicación, la administración del conocimiento y la necesidad de patrocinar y gestionar la diversidad, hacen necesario un entorno educativo que obligue permanentemente a las autoridades educativas a repensar las misiones, funciones y responsabilidades tradicionales de la educación (Lozoya, 2012). Cambios que son caracterizados por su vitalidad económica, es decir, por lo susceptibles que se encuentran a la globalización. Esto constituye el contexto en el que operan las Instituciones de Educación Superior (IES), con todos sus desafíos y oportunidades. Por lo que la importancia de la globalización se perfila por ser la etapa actual de la economía mundial que se caracteriza por las estrechas interrelaciones establecidas entre los países, hecho que tiene su base en el desarrollo alcanzado en los transportes, las comunicaciones, el procesamiento y transmisión automatizada de información. Sin embargo, este proceso no es un fenómeno exclusivamente tecnológico-económico, sus tendencias se presentan en todos los campos de la actividad humana de nuestros días (Plan de Estudios, 2010).

En adición, Contreras (2014) señala que el objetivo principal de las IES es la formación de profesionistas que atiendan a las demandas del país. El reto para la Universidad Nacional Autónoma de México (UNAM), es garantizar a los estudiantes que ingresan a sus aulas una formación de calidad que les permita ejercer como los mejores profesionales del campo, los más informados, responsables y críticos, es decir, una formación profesional que pueda responder a las necesidades destacadas de la sociedad.

Lo anterior plantea un desafío importante para las IES, pues deben enseñar a sus educandos el camino para ser analíticos y críticos, formándolos no sólo para el futuro, sino también para la complejidad del presente, pero teniendo claro que el porvenir será cada vez más complejo y no simplificará sus procesos; al contrario establecerá cada vez más interrelaciones que impliquen nuevos campos y métodos de formación (Garduño et al., 2008) que bien pueden ser expresados en diversos modelos educativos. 
El análisis de los modelos educativos está actualmente en el centro de las discusiones en torno al quehacer académico. Lo peculiar de esta discusión radica en el consenso cada vez más extendido de la necesidad de que las instituciones educativas cumplan satisfactoriamente la función de formar a las futuras generaciones en las competencias, capacidades y destrezas que requiere el desempeño laboral para una sociedad que se transforma profunda y rápidamente. Las instituciones educativas en la era del conocimiento giran alrededor de la promoción de competencias, que son las que pueden dar respuesta a las exigencias del mundo actual. Por su gran potencial como generadoras de desarrollo, las instituciones educativas deben asumir un compromiso con la transformación e innovación. Lo que implica una redefinición en sus programas y currículos. De esta manera las instituciones deben incrementar la calidad de los procesos de enseñanza-aprendizaje, y así responder a los requerimientos de las sociedades tales como: la globalización, economía y mercado laboral (Herrera et al., 2011). Es así como el interés por desarrollar competencias en el ámbito universitario tiene sus orígenes a finales de la década de los 60's y principios de los 70's. David McClelland, profesor de Psicología de la Universidad de Harvard, se dio cuenta de que los exámenes aplicados a los alumnos no eran capaces de prever el éxito o el fracaso laboral de los profesionales que egresaban, ya que era necesario también evaluar su desempeño al momento de resolver conflictos que el contexto les demandaba (Cerón, 2013).

En la incorporación de las competencias a los planes de estudio se encuentra latente el afán por desplazar el aprendizaje enciclopedista y memorístico producto de programas de estudio tradicionalistas, y por lo tanto, rígidos y ajenos a la realidad social. Se busca incorporar concepciones éticas y sociales fundamentadas en el conocimiento de las necesidades del contexto, que promuevan tanto el avance de la educación como de la sociedad. Las competencias están relacionadas con una tarea o actividad determinada. Son consecuencia de la experiencia y constituyen saberes articulados que una persona pone en marcha automáticamente.

La educación basada en competencias es una orientación educativa que pretende dar respuestas a las demandas y problemáticas sociales. Para Argudín (2005) el concepto de competencia, tal y como se entiende en la educación, resulta de las nuevas teorías de cognición y básicamente significa saberes de ejecución. Puesto que todo proceso de "conocer" se traduce en un "saber", entonces es posible decir que son recíprocos competencia y saber: saber pensar, saber desempeñar, saber interpretar, saber actuar en diferentes escenarios, desde sí y para los demás (dentro de un contexto determinado).

La Universidad Nacional Autónoma de México (UNAM) institución de educación superior líder en Latinoamérica, desde la década de los 70's como parte de sus políticas 
educativas, solicitó a sus entidades, la modificación de sus planes y programas de estudio, con la finalidad de actualizar sus contenidos, de que se elimine la rigidez curricular, se refleje la flexibilidad, y se incorporen perspectivas educativas centradas en el aprendizaje que favorezcan una formación de calidad en menor tiempo (preferentemente ocho semestres o su equivalente de cuatro años para los planes anuales). Con tal propósito los programas de estudios deberán ser congruentes con el contexto del país, de tal manera que el estudiante tenga los conocimientos, habilidades y actitudes que le permitirá desempeñarse con eficiencia en diversos ambientes, y que pueda realizar en menor tiempo sus estudios de pregrado, sin demerito de la calidad académica, además de que tendrá opciones de una formación flexible, acorde con sus intereses (Plan de Estudios, 2010).

Para esto, el plan de estudios de Psicología de la FES Zaragoza tiene como objetivo formar psicólogos generales con los conocimientos, habilidades, destrezas y actitudes necesarios que le permitan intervenir inter y multidisciplinariamente en individuos, grupos, organizaciones y comunidades, a partir de una formación integral, reflexiva y constructiva del entorno nacional y mundial, con fundamentos para aplicar el conocimiento, investigar sobre fenómenos relacionados con la disciplina, e innovar de acuerdo con el escenario en el que se desempeñen.

Lo cual se ve reflejado en su perfil de egreso, en donde el licenciado en Psicología es el profesional con una sólida formación, que posee los conocimientos, habilidades y actitudes para la promoción de comportamientos saludables; la prevención, intervención e investigación de diferentes problemas relacionados con la disciplina en diversos contextos y escenarios. Está capacitado para aplicar el conocimiento teóricometodológico de la disciplina y colaborar con otras en la solución de situaciones con problemas de índole psicológica, a nivel local, regional, nacional y mundial, relacionadas con la investigación, evaluación, diseño e implementación de programas de intervención para la prevención, orientación, rehabilitación y promoción (Plan de Estudios, 2010).

La problemática principal cuando se refiere al perfil de egreso se encuentra en que si bien un Plan de Estudios por competencias genéricas y disciplinares debe articular los conocimientos globales, conocimientos profesionales y experiencias laborales para que el estudiante sea capaz de reconocer las necesidades y problemas de la realidad. Se debe tomar en cuenta los requerimientos y dificultades de la sociedad; estas necesidades se definen mediante el diagnóstico de las experiencias de la realidad social, de la práctica de las profesiones, del desarrollo de la disciplina y del ámbito laboral. Esta combinación de elementos permitirá identificar las problemáticas hacia las cuales se orienta la formación profesional, para identificar y definir las competencias profesionales indispensables para el establecimiento del perfil de egreso del futuro profesionista. 
Para analizar las competencias profesionales de los egresados universitarios es necesario tener una visión clara del tipo de formación requerido por el mercado laboral. Las competencias profesionales de los trabajadores se definen como un conjunto identificable y evaluable de conocimientos, aptitudes, valores y habilidades relacionadas entre sí, que permiten desempeños satisfactorios en situaciones reales de trabajo, según estándares utilizados en el área ocupacional (Freire y Salcines, 2010). Por lo tanto, el momento de la titulación toma vital relevancia, como un puente entre las competencias que el estudiante ya ha desarrollado a lo largo de su formación académica, y el mercado laboral que extiende una serie de demandas profesionales; en ese momento, la obtención del grado es un momento crítico en el que el pasante tiene la posibilidad de fortalecer las competencias que ya pose, y también de adquirir otras más, de tal forma que el conjunto de estas le permitan responder a su quehacer profesional.

Lozoya (2012) sostiene que las competencias gerencias se encuentran implicadas en distintas ocupaciones o varios ámbitos del conocimiento; son apropiadas para la mayoría de las profesiones y están relacionadas con el desarrollo personal y la formación ciudadana. Describen comportamientos asociados al desempeño común de diversas ocupaciones y ramas de actividad productiva (analizar, planear, interpretar, negociar, etc.). Permite la organización y la agrupación de familias profesionales u ocupacionales. Se puede decir que las competencias genéricas identifican los elementos compartidos, comunes a cualquier titulación, como la capacidad para aprender, tomar decisiones y diseñar proyectos, así como las habilidades interpersonales, entre otras.

Al concluir exitosamente el Plan de Estudios con una motivación continúa generada por el interés del alumno en los conocimientos de la disciplina, aunado con la práctica enriquecedora entre pares y con el docente, sobresale la valoración positiva de la cantidad y calidad de los contenidos teóricos y prácticos del Plan de Estudios, así como la variedad y nivel de contenidos de especialización, se puede apostar en que la motivación constante en los alumnos, junto con un buen nivel del docente, generará una tendencia común a continuar la formación académica a través de posgrados y una elevada valoración en la elección del área de especialización, siendo la docencia y la investigación las áreas más demandadas por el estudiante egresado con alto rendimiento (Mercado-Ruiz, 2016).

El panorama anterior resulta en una dinámica donde si bien el foco principal es la adquisición de conocimientos en pro de una formación profesional de alta calidad, deja de lado elementos asociados a la rutina cotidiana del egresado, como lo puede ser los relacionados con el proceso de titulación, que de acuerdo a Martín et al (1999, como se citó en Escandell et al., 2001) los más relevantes se centran en las dudas sobre como afrontar la vida profesional, los cuestionamientos sobre la habilidad que se posee para la intervención, decisiones adyacentes a la propia etapa de desarrollo en la que se 
encuentra el egresado (búsqueda o consolidación de la pareja, crianza), aspiraciones y plan de vida.

El proceso de titulación de acuerdo con la Dirección General de Profesiones de la Secretaría de Educación Pública (SEP, 2018) representa la fase de culminación de los estudios que le permite al estudiante obtener un título y una licencia para ejercer su actividad profesional. Este proceso recupera los conocimientos, capacidades, habilidades, actitudes, valores y experiencias que desarrolló durante la carrera, los cuales se demuestran mediante diversas opciones y formas de evaluación; comúnmente denominadas "opciones de titulación".

Es de resaltar el hecho de que cada institución de educación superior reglamenta y estipula las opciones por medio de las cuales sus egresados pueden hacerse acreedores de un título universitario. En el caso de la UNAM, la titulación se conceptualiza como último proceso que "cuyos objetivos... son: valorar en conjunto los conocimientos generales del sustentante en su carrera; que este demuestre su capacidad para aplicar los conocimientos adquiridos y que posee criterio profesional". De acuerdo con el artículo 20 del Reglamento General de Exámenes de la UNAM, fueron aprobadas 10 opciones de titulación para que cada consejo técnico y comité académico seleccione las que a su conveniencia sean acorde a la formación de sus egresados. Por lo que las diez opciones se constituyen por: elaboración de TESIS, tesina, informe final de servicio social, reporte de trabajo profesional, examen general de conocimientos, ampliación y profundización de conocimientos, alto rendimiento y totalidad de créditos, estudios de posgrado, actividad de investigación y actividad de apoyo a la docencia (DGAEUNAM, 2011; UNAM, 2004).

En el caso particular de la Facultad de Estudios Superiores Zaragoza, el consejo técnico y el comité académico de carrera decidieron aprobar las 10 opciones sugeridas por la Dirección General de Administración Escolar, sin embargo, por su naturaleza y/o demanda, no todas las opciones han sido empleadas por egresados de la carrera. Ejemplo de ello es el caso de la titulación por estudios de posgrado, pues el mismo reglamento vigente, de acuerdo con su inciso H numeral I: Ingresar a una especialización, maestría o doctorado impartido por la UNAM, cumpliendo los requisitos correspondientes... uno de los requisitos básicos para ingresar a la mayoría de los posgrados en la UNAM es el título de licenciatura, lo cual limita sustancialmente la oferta y la demanda por esta opción. En la tabla 1 se observa el número de titulados por modalidad en la carrera de Psicología de la FES Zaragoza en el periodo de enero de 2015 a abril de 2020 . 
Tabla 1

Titulados por modalidad 2015-2020

\begin{tabular}{|c|c|c|c|c|c|c|}
\hline \multirow{2}{*}{\multicolumn{2}{|c|}{ Modalidad de Titulación }} & \multicolumn{4}{|c|}{ AÑO } & \multirow[b]{2}{*}{ 2019-2020 } \\
\hline & & 2015 & 2016 & 2017 & 2018 & \\
\hline \multicolumn{2}{|l|}{ Tesis } & 119 & 100 & 85 & 56 & 85 \\
\hline \multicolumn{2}{|l|}{ Tesina } & 14 & 14 & 6 & 7 & 3 \\
\hline \multicolumn{2}{|c|}{ Informe Final de Servicio Social } & 45 & 46 & 33 & 34 & 39 \\
\hline \multicolumn{2}{|c|}{ Seminario de Tesis/Tesina } & 0 & 0 & 0 & 0 & 0 \\
\hline \multicolumn{2}{|c|}{ Examen general objetivo } & 40 & 44 & 37 & 27 & 0 \\
\hline \multicolumn{2}{|c|}{ Total, de Créditos y Alto nivel académico } & 28 & 72 & 70 & 90 & 75 \\
\hline \multicolumn{2}{|c|}{ Informe de Trabajo Profesional } & 6 & 5 & 5 & 7 & 3 \\
\hline \multirow{3}{*}{$\begin{array}{l}\text { Ampliación y } \\
\text { profundización de } \\
\text { conocimientos }\end{array}$} & Diplomado & 39 & 80 & 107 & 78 & 104 \\
\hline & $\begin{array}{l}\text { Créditos } \\
\text { Adicionales }\end{array}$ & 16 & 60 & 74 & 53 & 59 \\
\hline & Cursos & 70 & 49 & 18 & 11 & 9 \\
\hline \multicolumn{2}{|c|}{ Actividad de Investigación } & 1 & 4 & 3 & 4 & 2 \\
\hline \multicolumn{2}{|c|}{ Apoyo a la Docencia } & 0 & 0 & 0 & 0 & 0 \\
\hline \multicolumn{2}{|l|}{ Posgrado } & 0 & 0 & 0 & 0 & 0 \\
\hline \multicolumn{2}{|l|}{ Total } & 378 & 474 & 438 & 367 & 379 \\
\hline
\end{tabular}

En esta tabla se puede observar claramente el dinamismo sobre la elección de los egresados respecto a qué opción de titulación es la más conveniente, si bien los números totales por año tienden a incrementarse de manera exponencial, no lo es así en cuanto el análisis individual por cada una de las opciones. Esta información lleva a suponer el hecho de que la titulación es un fenómeno dinámico que va más allá del mero ámbito académico, y donde además de involucrar factores asociados al rendimiento y competencias profesionales, factores personales, sociales y situacionales convergen en un proceso decisivo para el futuro profesionista, y sin su culminación, la vida académica pierde sentido.

Es por ello por lo que la noción de competencia cobra relevancia, ya que cada competencia se desarrolla a través de la articulación de habilidades cognitivas y prácticas, conocimiento, motivación, valores, aptitudes, emociones y otros componentes sociales y conductuales. Las competencias de los estudiantes son observables en la realidad cotidiana durante su proceso de titulación, y estas representan un trazo de unión entre las características individuales y las cualidades requeridas para llevar a cabo la obtención del grado. Existen múltiples y variadas definiciones en torno a la competencia académica y profesional. Un concepto generalmente aceptado la define como la 
capacidad efectiva para llevar a cabo exitosamente una demanda contextual plenamente identificada. Asimismo, la competencia profesional puede ser una probabilidad de éxito en la ejecución del trabajo, también es una capacidad real y demostrada de saber hacer las cosas. Además, es una habilidad productiva del individuo, que se define y se mide en términos de ejecución en un determinado contexto profesional o académico, y no solamente de conocimientos, habilidades, destrezas y aptitudes; éstas son necesarias, pero no suficientes por sí mismas para el desempeño efectivo de un trabajo. Ya que el éxito radica en la articulación de estos elementos en su conjunto, y no en su forma aislada. Si bien, se ha puesto especial interés en investigar el desarrollo de competencias durante la formación académica del estudiante, es de vital importancia no desdibujar el proceso de titulación como un momento crucial en el que el estudiante fortalece sus competencias adquiridas e incluso desarrolla nuevas.

Por lo anterior este estudio se divide en dos etapas, la primera tiene por objetivo el identificar las categorías o indicadores que integran las competencias profesionales en el proceso de titulación de los recién egresados de la carrera de Psicología. La segunda etapa, proyecta el utilizar la información resultante como a base para el desarrollo de una medida de evaluación pertinente sobre las competencias profesionales vinculadas al proceso de titulación.

\section{Método}

Para fines de este estudio, el procedimiento general se dividió en dos etapas. La primera con el objetivo de identificar a partir de la teoría y la evidencia empírica, las categorías y subcategorías que integran las competencias que tanto estudiantes de últimos semestres como egresados, aplican a su proceso de titulación. La segunda etapa consistió en la construcción de la Escala de Competencias aplicadas a la Titulación, esto al determinar sus indicadores psicométricos.

Etapa 1: Exploración de las competencias profesionales vinculadas al proceso de titulación

\section{Participantes}

Por medio de una muestra de casos-tipo se contó con la participación de 20 egresados, 10 hombres y 10 mujeres recién titulados de la carrera de Psicología (Titulación $=8$ meses). Obtuvieron el grado de Licenciatura por las siguientes modalidades: 11 mediante prueba escrita (actividad de investigación: tesis, tesina y publicación de artículo de investigación; o informe final de servicio social y/o trabajo profesional); y 9 por prueba no escrita (examen profesional objetivo y ampliación-profundización de 
conocimientos: diplomado, cursos o créditos adicionales). El rango de edad fue de 23 a 26 años (Medad = 25.7 años), solteros, sin hijos y en su mayoría (13 participantes) realizaban alguna actividad laboral vinculada con la Psicología. La duración de sus estudios fue de 4.2 años, con un promedio académico de 8.7. Todos los participantes cubrieron con los requisitos de titulación: $100 \%$ de los créditos de la carrera, servicio social y aprobación de idioma inglés, estos dos últimos, durante el primer año de egreso (solo dos de los participantes tardaron más de un año en presentar su servicio social). En lo referente a su proceso de titulación, este tuvo una duración promedio de 18.9

meses y al momento de tomar la decisión sobre por cuál modalidad optarían para titularse, tenían una noción básica respecto a requisitos y procesos administrativos en promedio, de 6 distintas modalidades de las 11 aprobadas por el $\mathrm{H}$. Consejo Universitario (DGAE-UNAM, 2011).

Tipo de Estudio y técnica de análisis de datos

Para esta etapa se consideró un enfoque cualitativo, debido a que él objetivo planteado, buscaba identificar el significado y estructura dinámica del fenómeno y que da razón plena de los comportamiento y manifestaciones" (Martínez, 2006; Vasilachis, 2006) esperados; en este caso, lo que los participantes pudieran expresar de sus competencias profesionales aplicadas a su proceso de titulación, así como las actitudes hacia el mismo. Por lo tanto, se trató de un estudio exploratorio, cuya visión general mediante el análisis de los datos, permite una aproximación a los elementos que componen el fenómeno mediante la exploración de sus dimensiones, categorías e indicadores mediante codificación cerrada (Álvarez- Gayou, 2003; Herrera, 2008; Sabino, 2000).

\section{Instrumento y técnica de recolección de datos}

Como técnica de recolección de datos se utilizó la entrevista semiestructurada de forma individual, en la cual se aplicó un cuestionario sociodemográfico y 30 cuestionamientos abiertos, que indagaban 5 dimensiones: los conocimientos de los participantes sobre el proceso de titulación, sus motivaciones respecto a la obtención del grado, su percepción sobre competencias profesionales adquiridas y desarrolladas durante el proceso, las limitantes que enfrentaron para titularse, y sus redes de apoyo.

\section{Procedimiento}

Se localizó a los participantes por medio de los registros de titulación de los últimos 3 meses al momento de este estudio. Los datos de contacto fueron proporcionados por la Secretaría Técnica de la Carrera de Psicología previa autorización de su jefatura. Con esto, se invitó vía correo electrónico y de manera personal a cada participante. Una vez realizado el contacto, se les explico el objetivo de la investigación 
y procedimiento. Se proporcionó una carta de consentimiento informado, garantizando confidencialidad y anonimato de la información proporcionada. Las entrevistas se realizaron con un promedio de duración de 40 minutos.

Las trascripciones de las entrevistas se realizaron mediante el procesador de textos Microsoft Office Word 365 y se utilizó el programa para análisis de datos cualitativo Atlas ti (versión 7). Para el análisis de los resultados se utilizó la codificación cerrada con el objetivo de categorizar la información relevante y susceptible a contraste teórico. El proceso se llevó a cabo en cuatro etapas: Codificación cerrada de la información (abstracción de los datos); Categorización; Creación de red semántica sobre relaciones entre las categorías, y Conformación de hallazgos teóricos.

\section{Resultados}

Después de realizar el análisis de indicadores extraídos de las entrevistas, se obtuvieron 6 categorías y 25 subcategorías que a continuación se describen.

La primera categoría fue nombrada Motivos para titularse se explica por indicadores como la rapidez del trámite, la posibilidad de profundización en los conocimientos que ofrece la modalidad de titulación, la conveniencia de acuerdo con gusto/interés del egresado y la relación existente con las actividades actuales.

La categoría dos descrita como las Competencias previas que tenían los egresados antes de iniciar su proceso de titulación; conocimientos teóricos, habilidades para la investigación, conocimiento de la titulación y competencias valorativas sobre el proceso, son sus indicadores.

En cuanto a la categoría tres, denominada Competencias adquiridas durante la titulación, se encontró como parte de sus indicadores, las competencias metodológicas y valorativas aprendidas durante el proceso, el acercamiento al mundo laboral, el manejo del tiempo, continuación de la formación para el desarrollo personal, búsqueda de una especialización teórica y sentido de la relación teoría-práctica.

La cuarta categoría se nombró Atribución a la titulación, la cual se explica por elementos personales como el compromiso y disposición para el estudio, el sentido de superación personal y profesional, y el vínculo con las redes de apoyo (familia, profesores, tutor, pacientes/participantes y amigos).

Por otro lado, también se registraron elementos externos como el plan de estudios cursado, el empleo actual y la obtención del pergamino.

La categoría cinco fue definida como Satisfacción al titularse, la cual es consecuencia de haber logrado avanzar por objetivos, el aprendizaje obtenido durante el proceso, la opción de titulación elegida, e incluso, la atención administrativa recibida. 


\section{Figura 1}

Red Semántica de categorías y dimensiones de competencias y actitudes aplicadas al proceso de titulación

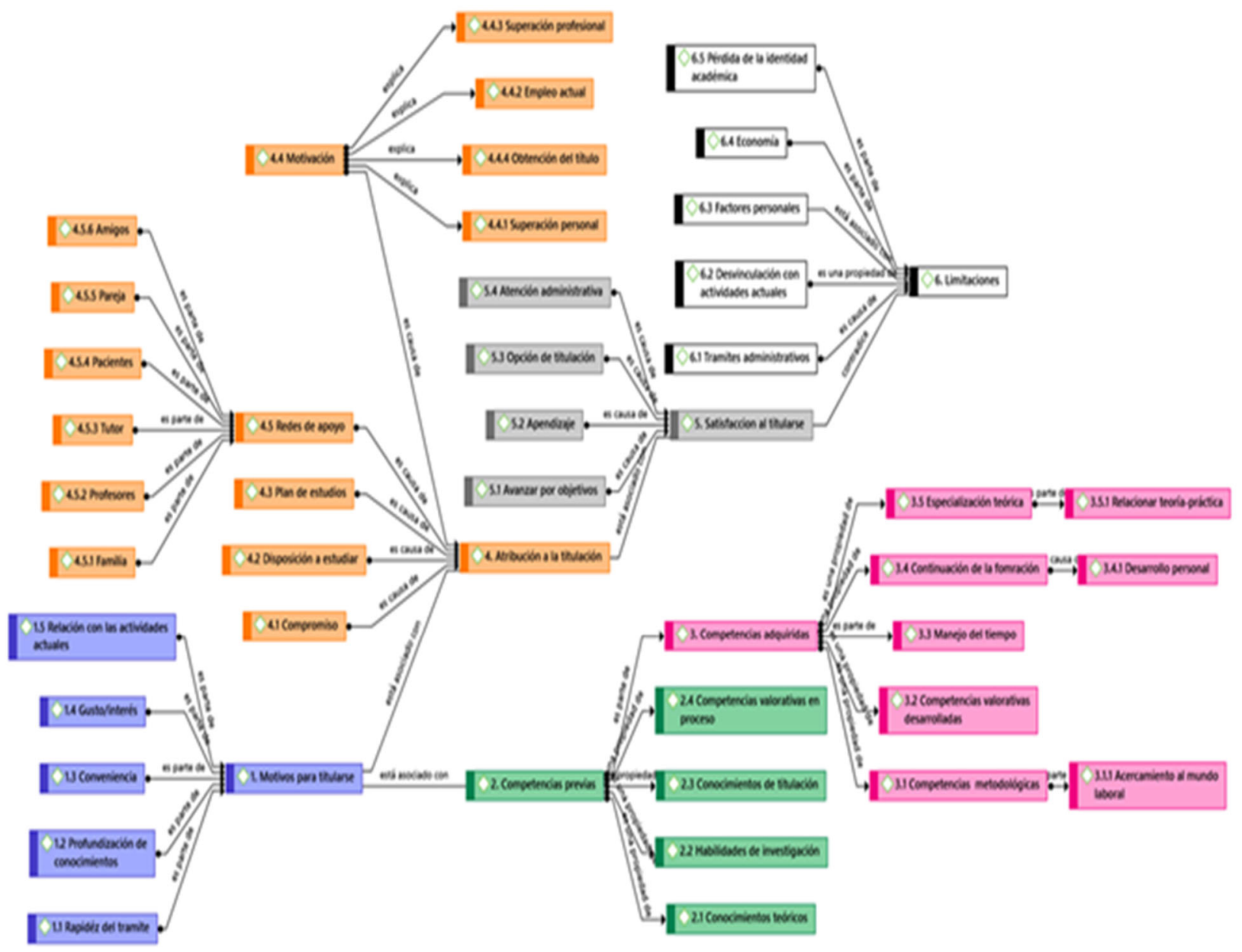

Finalmente, la sexta categoría hace alusión a las Limitaciones que, de acuerdo con los participantes, dificultaron su proceso de titulación: trámites administrativos, desvinculación de la modalidad de titulación con las actividades actuales, factores personales, economía y pérdida de identidad académica.

En la figura 1 se presenta la red semántica en la que se visualiza la relación hallada entre dimensiones y categorías. Se puede identificar que los motivos para titularse se asocian con las competencias previas a iniciar el proceso de titulación del egresado, estas competencias son precursoras para las que se pueden adquirir dúrate el proceso. Por otro lado, los motivos para titularse se asocian tanto con la atribución como la satisfacción que el egresado da al proceso; y estos a su vez, se presentan como contrarios a las limitantes que pudieran identificarse durante. 
Etapa 2: Construcción de la Escala de Competencias aplicadas a la Titulación

\section{Participantes}

Para esta segunda etapa se contó con la participación de 434 estudiantes del último semestre de la carrera de Psicología, pertenecientes a la generación 2015-2018 ( $76 \%$ mujeres, $24 \%$ hombres), quienes fueron seleccionados por medio de un muestreo no probabilístico intencional. El rango de edad oscilo entre los 20 a 39 años (Medad=22.1 años). 93.1\% se declaró como soltero y el 5.5\% tenía hijos al momento del estudio. Únicamente el $70.3 \%$ era estudiante de tiempo completo, mientras el resto tenía una ocupación que variaba de acuerdo con lo siguiente: $8 \%$ estudiantes con trabajo relacionado con la psicología, $20.3 \%$ estudiantes con trabajo NO relacionado con la psicología y $1.4 \%$ se desempeñaba como becario o realizando prácticas profesionales. En cuanto a su trayectoria académica, la tabla 2 muestra el orden en que cursaron sus estudios entre las cuatro áreas que tienen como opción de elegir a lo largo de la carrera.

\section{Tabla 2}

Trayectoria académica de los estudiantes

\begin{tabular}{lllll}
\hline $\begin{array}{l}\text { Área por } \\
\text { cursar } \\
\text { Semestre }\end{array}$ & $\begin{array}{l}\text { Psicología } \\
\text { Educativa }\end{array}$ & $\begin{array}{l}\text { Psicología } \\
\text { Clínica y de } \\
\text { la Salud }\end{array}$ & $\begin{array}{l}\text { Psicología } \\
\text { Social }\end{array}$ & $\begin{array}{l}\text { Psicología del } \\
\text { Trabajo y las } \\
\text { Organizaciones }\end{array}$ \\
\hline $3^{\mathrm{o}}$ y $4^{\circ}$ & $44.9 \%$ & $12.7 \%$ & $19.6 \%$ & $22.8 \%$ \\
$5^{\mathrm{o}}$ y $6^{\circ}$ & $24.9 \%$ & $53.5 \%$ & $12 \%$ & $9.7 \%$ \\
$7^{\mathrm{o}} \mathrm{y} 8^{\circ}$ & $18.2 \%$ & $25.8 \%$ & $24.9 \%$ & $30.9 \%$ \\
\hline
\end{tabular}

Nota. Primer y segundo semestres pertenecen al tronco común "Etapa Básica", los alumnos a partir de tercer semestre pueden elegir entre una de las cuatro áreas ofertadas para cursar cada año.

El promedio general fue de 8.99 y en lo que respecta a los requisitos de egreso, el $7.6 \%$ de los participantes habían cubierto el servicio social y el $28.3 \%$ aprobado la comprensión del idioma inglés como lengua extranjera, con lo cual cumplen con los requisitos mínimos para iniciar su proceso de titulación inmediatamente terminen su semestre lectivo. Sin embargo, 9.2\% de los participantes adeudaban de 1 a 2 materias y $4.6 \%$ más de 3 . Por último, el $67.5 \%$ pretende iniciar tramites de titulación en no más de seis meses después de terminar el $100 \%$ de créditos de su plan de estudios, donde las preferencias en su elección de modalidad de titulación son: $42.9 \%$ se inclina por una opción de titulación mediante prueba escrita (actividad de investigación: tesis, tesina y 
publicación de artículo de investigación; o informe final de servicio social y/o trabajo profesional), 36.4\% por una opción de prueba no escrita (ampliación-profundización de conocimientos: diplomado, cursos o créditos adicionales), $15.7 \%$ buscaría titularse totalidad de créditos y alto rendimiento académico (promedio mayor a 9.5), y solo el $5.1 \%$ aún no se decide. Las opciones de titulación de examen profesional objetivo, posgrado y apoyo a la docencia no se reportaron.

\section{Instrumento}

Con base a los resultados de la etapa anterior, se diseñó un cuestionario de auto aplicación dicotómico de 66 ítems cuyas opciones de respuesta determinan el dominio o no dominio de diversas competencias aplicadas al proceso de titulación, así como las actitudes hacia el mismo. El cuestionario además incluye un apartado de datos sociodemográficos y académicos. Los ítems fueron distribuidos en 6 dimensiones (Motivación, Competencias previas, Competencias Adquiridas, Atribución, Satisfacción y Limitantes) cada una constituida por 11 reactivos que fueron evaluados mediante juicio de expertos.

\section{Procedimiento}

Por tratarse de un estudio psicométrico de una sola muestra aleatoria simple, los participantes fueron localizados en las aulas dentro de su horario académico durante ambos turnos escolares. La aplicación fue grupal y únicamente después leerles el consentimiento informado y aceptar participar se les entrego el instrumento. Una vez recabados los datos fueron capturados en el programa SPSS v.24 para su análisis.

\section{Resultados}

En un primer momento, los 66 ítems se sometieron a la evaluación de 10 expertos en temas relacionados con formación profesional, diseño curricular, campo laboral y procesos académico-administrativos. El jueceo consistió en valorar la suficiencia, coherencia, relevancia y claridad de cada ítem respecto a la dimensión en la que se encontraba. El análisis de validez de contenido mediante el coeficiente de concordancia Kappa de Cohen arrojo un valor total $k=.94$ para 58 de los 66 ítems. Se eliminaron los reactivos $3,23,24,30,55,56,61$ y 66 .

Posteriormente y en función de los resultados obtenidos se aplicó un análisis de discriminación para grupos extremos y consistencia interna, con lo que el instrumento fue reducido de nueva cuenta a 53 reactivos, eliminando así, los ítems 5, 11, 16, 32 y 47 por no cubrir los mínimos necesarios en discriminación $(\mathrm{t}=\mathrm{p}$.> .05) o consistencia en rangos de .70 a .90. Finalmente se empleó un análisis de confiabilidad por método Split 
half de $\mathrm{R}=.681$ en su coeficiente de Spearman-Brown para longitudes desiguales, con lo que el cuestionario terminó conformándose de 49 reactivos dicotómicos agrupados en las 6 dimensiones propuestas.

Los coeficientes de correlación Spearman-Brown, el número de reactivos y la definición por dimensión son las siguientes:

Dimensión 1: Motivación $\mathrm{R}=.615$ (reactivos 1, 9, 16, 22, 41). Se define como las razones y circunstancias que propiciarían la toma de decisiones para optar por una opción de titulación específica. Contempla indicadores como: rapidez, profundización de conocimientos, conveniencia, preferencia y relación con las actividades actuales.

Dimensión 2: Competencias Previas $\mathrm{R}=.702$ (reactivos 2, 7, 10, 28, 42). Se entiende como aquellas competencias dominadas previamente al inicio del proceso de titulación, y que se identifican como necesarias con el fin de desarrollar o afianzar competencias específicas de la opción de titulación elegida. Contempla indicadores como: dominio de teorías, modelos y conceptos psicológicos, habilidades metodológicas para la investigación, valores para la autorregulación y conocimiento de los procesos administrativos de titulación.

Dimensión 3: Competencias Adquiridas $\mathrm{R}=.600$ (reactivos 3, 11, 17, 23, 29, $33,36,44)$. Hace referencia a las competencias específicas que se pueden desarrollar durante el proceso de titulación de acuerdo con la opción escogida. Se compone por indicadores como: competencias metodológicas y valorativas, especialización teórica, identificación de la relación teórico-práctica, administración del tiempo, acercamiento al mundo laboral y vínculo con la formación continua.

Dimensión 4: Atribución $\mathrm{R}=.524$ (reactivos 4, 5, 12, 18, 19, 21, 2426, 30, $37,38,40,42,47,48,49)$. Se trata de la interpretación del estudiante sobre las causas y razones sobre el éxito en su proceso de titulación. Compromiso y motivación personal, disposición al estudio autorregulado, redes de apoyo, vínculo con el empleo o actividades actuales, necesidad de desarrollo y superación profesional, y encausamiento del plan de estudios cursado son ejemplo de sus indicadores.

Dimensión 5: Satisfacción $\mathrm{R}=.511$ (reactivos 6, 13, 25, 39). Se entiende como la sensación de bienestar propio, producto de indicadores como el avanzar por objetivos, obtención de nuevos aprendizajes o conocimientos, opción de titulación elegida y percepción del trato administrativo durante el proceso.

Dimensión 6: Limitantes $\mathrm{R}=.569(8,14,15,20,2731,32,35,46)$. Se define como las fronteras autoimpuestas por el estudiante que dificultarían su proceso de titulación. Contempla indicadores como: complejidad de los tramites 
administrativos, desvinculación de las actividades actuales y la opción de titulación, economía, perdida del rol de estudiante, indecisión, desinterés, incertidumbre y desvalorización profesional.

Finalmente, se presenta un ejemplo de los ítems que conforman la Escala de Competencias y Actitudes aplicadas a la Titulación (ECAT).

Ejemplo de reactivos que conforman la ECAT

Dimensión 1: Motivación

1. El proceso de titulación debe de ser un trámite rápido.

9. La opción de titulación que elija me ayudara a profundizar más en un tema determinado.

16. Es irrelevante la manera en que me titule, lo que importa es que lo haga.

Dimensión 2: Competencias Previas

28. Tengo los suficientes conocimientos teóricos para poder desarrollar un tema de investigación.

2. Estoy familiarizado con el uso del formato APA para desarrollar una investigación.

10. Conozco el proceso administrativo de las distintas opciones de titulación.

Dimensión 3: Competencias Adquiridas

23. Puedo apegarme a un horario específico para trabajar en mí proceso de titulación.

33. El proceso de titulación me dio herramientas para continuar formándome como psicólog@.

43. A través de mi proceso de titulación logre relacionar la teoría con la práctica profesional.

Dimensión 4: Atribución

5. El titularme es un compromiso al que esta prohibido fallar.

46. He planificado las opciones que tengo para titularme.

36. Tengo disposición para estudiar.

Dimensión 5: Satisfacción

38. Se que durante mí proceso de titulación aprenderé algo nuevo.

6. Algunas opciones de titulación son mejores que otras.

25. La atención administrativa que recibiré durante mí proceso de titulación será la ideal. 


\section{Dimensión 6: Limitantes}

20. Los trámites administrativos dificultan el proceso de titulación.

41. Mis actividades actuales están totalmente desvinculadas de la forma en la que pretendo titularme.

14. El dejar de ser alumno complicaría que pueda titularme.

\section{Discusión}

Con los resultados obtenidos de la etapa 1 se puede observar que la titulación representa un fenómeno multivariado, tal y como lo menciona Alonso-Martín (2010). Los egresados identifican los elementos del proceso de titulación, considerando que hay un cambio positivo entre el antes y después de titularse, tanto a nivel personal como en cuanto a sus herramientas. El tener un título los conecta con el ámbito laboral, mejora su percepción sobre sus posibilidades de empleo, los hace capaces de obtener conocimientos y aplicarlos en su formación profesional, asimismo los motiva para estudiar un posgrado. En esta etapa se encontró que la elección de la opción de titulación es significativa porque representa un paso hacia la consolidación profesional de la vida adulta, referido por Freire y Salcines (2010). El tiempo jugó un papel esencial en la obtención del título, debido a que limitó terminar con antelación sus proyectos académicos, la mayoría de ellos coincide en que pudieron realizar sus trámites en un menor lapso si hubieran contado con un mejor manejo de su vida personal, lo cual posibilitaría el elegir de manera más objetiva la mejor opción de acuerdo a su plan de vida, el trato por parte de la administración escolar fuera empático y cordial, así como acortar los trámites administrativos.

Por otro lado, y de acuerdo con los resultados expuestos en la etapa dos de este estudio, se puede determinar que se logró el objetivo de construir un instrumento que permita evaluar la percepción de los estudiantes sobre su empleo de competencias y actitudes hacia su proceso de titulación, de manera exitosa. El cuestionario diseñado contempla las seis dimensiones planteadas inicialmente en la etapa uno de este estudio. A modo de que las competencias previas al proceso de titulación, las competencias adquiridas durante el proceso, la motivación para titularse, la satisfacción que representa el titularse por alguna opción en específico, la atribución que se le da al poder titularse y las limitantes que se perciben respecto al proceso, fueron mantenidas a pesar de someterse al proceso estadístico de validez y confiabilidad

En lo que respecta al proceso de conformación de los reactivos del cuestionario, se evidencian tres elementos a considerarse relacionados al fenómeno que se pretendió evaluar: 1) coherencia teórica entre la definición de competencias y el cómo se pueden evaluar (Alonso-Martín, 2010; Suárez, 2011); 2) el optar por una evaluación dicotómica 
en lugar de otra como un moderador de la diversidad/supuestos teórico-prácticos de las competencias, además de poder incluir la riqueza de elementos actitudinales del proceso (Domínguez, 2013); y 3) se tomaron las decisiones ad hoc respecto a la eliminación de reactivos no por inclinación o especulación de los investigadores, sino por un proceso estadístico riguroso y estandarizado para instrumentos como el que se desarrolló.

Por otro lado, en cuanto al análisis de consistencia del instrumento por medio del procedimiento de Split-half y cuyo valor es $\mathrm{R}=.681$, se denota una confiabilidad aceptable con tendencia a optima, lo que asegura la utilidad del instrumento a lo largo del tiempo y en distintos contextos académicos. Si bien es necesario asegurarse de su estabilidad a lo largo de periodos de tiempo razonables (por lo menos una reevaluación de sus propiedades psicométricas, incluyendo la validez dentro del próximo par de años) se considera factible y necesario el hacer uso de otros elementos que reafirmen la efectividad del cuestionario. Elementos que lo lleven a contrastarse con medidas parales o algún indicador de criterio interno u externo que pongan a prueba dicha consistencia (Nunally, 1978).

Bajo esta línea, la validez de expertos obtenida evidencia la solidez de la base teórica sobre la cual se desarrolló el cuestionario. Si bien al inicio se planteó un número mayor de reactivos por dimensión, la eliminación por acuerdo de los expertos enfatiza que los ítems restantes son representativos de un fenómeno fundamentado no tan solo en la teoría, sino que en la praxis es identificable y susceptible a evaluación. Claro esta que esta versión reportada del instrumento debe de someterse a su análisis confirmatorio en pro de llevar a un estándar mucho mayor la validez de esta herramienta.

Respeto a las dimensiones de competencias tanto en su perspectiva de las previas a iniciar el proceso de titulación, así como las adquiridas durante, fungen como indicadores concretos de la efectividad en la formación del plan de estudios que los alumnos cursaron. Si bien no se puede generalizar el que los indicadores que conforman estas dimensiones sean la totalidad de competencias necesarias y/o empleadas en todos los procesos de titulación, si permiten echar un vistazo superficial a las habilidades o herramientas consideradas competencias mínimas que todo egresado debería de manejar al momento de emprender esta etapa final de su vida académica. Como lo menciona Ortega y Zych (2013), se espera que el egresado cuente ya con las cualidades de un profesionista específico en virtud de evaluar e intervenir desde lo individual a lo comunitario en fenómenos socialmente relevantes.

Las dimensiones de motivación, atribución y satisfacción respecto a la titulación proporcionarían entonces la información necesaria para comprender el porqué de los alumnos que emplean o ejecutan en distinta medida cada una de las competencias requeridas para el titularse (por lo menos en este estudio, ya que también puede aplicar en otras circunstancias académico-profesionales). Esto puede ser atribuido a que son 
factores mediadores del rendimiento del egresado. Su visión de qué tan eficaz o no puede ser su proceso de titulación, más allá de qué tan hábil se considera en el uso de ciertas habilidades, gira entorno a lo que percibe como compromiso, disposición, facilitadores externos, facilidad del trámite, nuevos aprendizajes, avanzar por objetivos, entre otros indicadores de las dimensiones señaladas. Esta perspectiva abre el campo a un proceso de orientación empático con los egresados, eficaz con vías a impactar la eficiencia terminal (Hernández et al., 2013) y sobre todo, una orientación académica que sobrepase la barrera del currículo oculto.

Sin embargo, una de las limitantes en este proceso de desarrollo del cuestionario, se focaliza en la poca o nula información empírica sobre el tema de la titulación, ya que como lo señalan Ortega y Zych (2013) lo que resulta alarmante, ya que, si bien existe todo un campo de investigación educativa, que puede ir desde el desempeño académico, la deserción, la reprobación, diseño y evaluación curricular, interacción en ambientes educativos y un sin fin de variables atribuibles a sus actores y ambientes académicos, el proceso de titulación se deja de lado, como un simple indicador o resultado de un plan de estudios. Dejar de lado la riqueza de información que puede proporcionar esta etapa aún académica, conlleva a tener evaluaciones amputadas y diagnósticos refutables sobre la formación profesional, en nuestro caso en particular, en Psicología.

Por último, hay que enfatizar que este cuestionario es un primer acercamiento a la evaluación del proceso de titulación como un aliciente de la formación profesional. Las propiedades psicométricas son las idóneas para ser utilizado en ambientes académicos y con fines de investigación, sin embargo, está claro que se necesita verificarse continuamente respecto a otras variables de investigación de relevancia, así como el someterse a diversos estudios donde el foco sean aquellos factores adyacentes al proceso de titulación. Lo anterior con el fin de probar su sensibilidad como herramienta de evaluación y de investigación, ya que el mismo proceso de titulación es un campo poco explorado y que requiere de atención urgente. En conclusión, la formación profesional y la titulación pueden ser concebidos como un enlace fértil para la delimitación de perfiles que ayuden en la toma de decisiones tanto en el diseño curricular, la eficiencia terminal, el abatimiento del rezago académico y la introducción formal al campo laboral.

Por último, se espera que estos resultados aporten información relevante al campo del diseño curricular, al ser la base que permita la vinculación del perfil de egreso de los estudiantes con estrategias didácticas encaminadas a fortalecer sus competencias, la toma de decisiones y el proceso de acreditación de la carrera. 
Agradecimientos

Esta investigación se concretó gracias al Programa de Apoyo a Proyectos para la Innovación y Mejoramiento de la Enseñanza, de la Dirección General de Asuntos del Personal Académico, Universidad Nacional Autónoma de México

\section{Referencias}

Alonso-Martín, P. (2010). La valoración de la importancia de las competencias transversales: comparación de su percepción al inicio y final de curso en alumnos de psicología. Revista de Investigación Educativa, 28(1), 119-140.

Álvarez- Gayou, J. (2003). Cómo hacer investigación cualitativa. Fundamentos y metodología. Paidós.

Argudín, Y. (2005). Educación basada en competencias. Nociones y antecedentes. Editorial Trillas.

Cerón, J. S. (2013). Modelo educativo basado en competencias. Trillas.

Contreras, M. (2014). Formación en psicología: una propuesta. Universidad Nacional Autónoma de México.

Dirección General de Administración Escolar, Universidad Nacional autónoma de México (DGAE, UNAM) (2011). Opciones de titulación en la UNAM. Universidad Nacional autónoma de México.

Domínguez, S. (2013). ¿Ítems Polinómicos o Dicotómicos? Un estudio empírico con una escala unidimensional. Revista Argentina de Ciencias del Comportamiento, 5(3), 30-37. http://revistas.unc.edu.ar/index.php/racc/

Escandell, M., Marrero, G., Castro, J. y Rodríguez, A. (2001). El abandono universitario: un estudio de la deserción en las titulaciones de Maestro y Educación Social. Anuario de Filosofía, Psicología y sociología, 4, 81-93.

Florentino, E. (2001). Sociedad de la información y educación. Consejería de Educación, Ciencia y Tecnología.

Freire, M. y Salcines, V. (2010). Análisis de las competencias profesionales de los titulados universitarios españoles. La visión de los egresados: Perfiles educativos, 32(130), 103-120.

Garduño, T., Guerra, M. E., y Sanchez, S. (2008). Una educación basada en competencias. Aula Nueva.

Hernández, Y., Martínez, J. y Carranza, C. (2013). Programa Institucional de Tutorías: Una estrategia para elevar el nivel de aprovechamiento en una institución educativa. Acta universitaria, 23(1), 31-36. 
Herrera, J. (2008). La investigación cualitativa. https://juanherrera.files.wordpress.com/2008/05/investigacion-cualitativa.pdf

Herrera, L., Jiménez, G. y Castro, A. (2011). Aprendizaje del alumno universitario de primer y último curso de las titulaciones de Psicología y Magisterio. Electrónic Journal of Research in Educational Psychology, 9(2), 659-692.

Lozoya, E. (2012). ¿Como implementar y evaluar las competencias genericas? Limusa.

Martínez, M. M. (2006). La investigación cualitativa (síntesis conceptual). Revista IIPSI. $9(1)$

http://sisbib.unmsm.edu.pe/bvrevistas/investigacion_psicologia/v09_n1/pdf/a09v9 n1.pdf

Mercado-Ruíz, A. (2016). Desarrollo de competencias del estudiante de psicología en FES Zaragoza [Tesis de Licenciatura no publicada]. Facultad de Estudios Superiores Zaragoza, Universidad Nacional Autónoma de México.

Nunnally, J. (1978). Teoría psicométrica. McGraw-Hill

Ortega, R. y Zych, I. (2013). Profesionales de la psicología y formación universitaria: buscando referencias para el título de Grado en Psicología. International Journal of Psychology and Psychological Therapy, 13(1), 83-96.

Plan de Estudios. (2010). Plan de estudios 2010 de la licenciatura en Psicología (Sistema Presencial) Aprobado por el Consejo Académico de Área de Ciencias Biológicas, Químicas y de la Salud, el 28 de septiembre de 2010, Acuerdo número 2/III/10. México: Facultad de Estudios Superiores Zaragoza, Universidad Nacional Autónoma de México.

Sabino, C. (2000). El proceso de la investigación. Enapo.

Secretaría de Educación Pública (2018). Autorización y registro profesional. https://www.sep.gob.mx/es/sep1/sep1_Registro_de_Titulo_y_Ex_cedula

Suárez, X. (2011). Valoración de las Competencias de Psicología: Estudio exploratorio en muestras de Estudiantes y Profesionales Activos. Revista de Psicología, 20(1), 73-102.

Universidad Nacional Autónoma de México (2004). Reglamento General de Exámenes. http://abogadogeneral.unam.mx/PDFS/COMPENDIO/170-2.PDF

Vasilachis, G. (2006). Estrategias de investigación cualitativa. Gedisa. 